\title{
EDITORIAL
}

\section{Preface: High Energy Astrophysics}

\author{
Bing Zhang ${ }^{1,2, *}$, Peter Mésáros ${ }^{3, \dagger}$ \\ ${ }^{1}$ Kavli Institute for Astronomy and Astrophysics and Department of Astronomy, Peking University, China; \\ ${ }^{2}$ Department of Physics and Astronomy, University of Nevada, Las Vegas, USA \\ ${ }^{3}$ Department of Astronomy and Astrophysics, Department of Physics, and Center for Particle and Gravitational Astrophysics, \\ Pennsylvania State University, University Park, PA 16802, USA \\ E-mail: *zhang@physics.unlv.edu, ${ }^{\dagger} n n p @ a s t r o . p s u . e d u$ \\ Received May 24, 2013; accepted May 29, 2013
}

\begin{abstract}
High energy astrophysics is one of the most active branches in the contemporary astrophysics. It studies astrophysical objects that emit X-ray and $\gamma$-ray photons, such as accreting super-massive and stellar-size black holes, and various species of neutron stars. With the operations of many space-borne and ground-based observational facilities, high energy astrophysics has enjoyed rapid development in the past decades. It is foreseen that the field will continue to advance rapidly in the coming decade, with possible ground-breaking discoveries of astrophysical sources in the high-energy neutrino and gravitational wave channels. This Special Issue of Frontiers of Physics is dedicated to a systematic survey of the field of high energy astrophysics as it stands in 2013.
\end{abstract}

PACS numbers 95.85.Nv, 95.85.Pw, 95.85.Ry, 95.85.Sz, 97.60.Lf, 97.60.Jd, 97.80.Jp, 98.62.Js

High energy astrophysics is the branch of astrophysics that studies astrophysical objects that emit high energy photons (X-rays and $\gamma$-rays), and more generally, emit non-thermal photons outside the traditional optical wavelengths. By studying non-thermal emission in the universe, one can unveil the part of universe that is not in a steady state, usually originating in violent environments near compact objects, such as neutron stars and black holes of different scales. These objects, besides emitting the broad-band non-thermal electromagnetic radiation, are also believed to be emitters of other signals outside the electromagnetic channel. These multi-messenger signals include cosmic rays, neutrinos, and gravitational waves. The field of high energy astrophysics, along with cosmology and planetary sciences, is one of the three most active branches in the contemporary astrophysics.

During the past decades, the field of high energy astrophysics has enjoyed a rapid development, thanks to an impressive list of space-borne and ground-based observational facilities. The currently operating space missions dedicated to high energy astrophysics include the Chandra X-ray Observatory, the X-ray Multi-Mirror Mission (XMM)-Newton, Suzaku, and the Nuclear Spectroscopic Telescope Array (NuSTAR) in the X-ray band; Swift and Integral in the hard X-ray/soft $\gamma$-ray band; the
Fermi Gamma-Ray Space Telescope and Astro-rivelatore Gamma a Immagini LEggero (AGILE) in the hard $\gamma$ ray band; as well as Cosmic Ray Energetics and Mass (CREAM) and Alpha Magnetic Spectrometer Experiment (AMS-02) cosmic ray detectors. On the ground, high energy facilities include the TeV telescopes High Energy Stereoscopic System (H.E.S.S.) and Very Energetic Radiation Imaging Telescope Array System (VERITAS); cosmic ray detectors Pierre Auger Observatory, Telescope Array and Astrophysical Radiation with Groundbased Observatory at YangBaJing (ARGO-YBJ); high energy neutrino detectors the IceCube Neutrino Telescope, Astronomy with a Neutrino Telescope and Abyss environment RESearch (ANTARES) and Cubic Kilometre Neutrino Telescope (KM3NeT); as well as gravitational wave detectors Laser Interferometer Gravitational Wave Observatory (LIGO) and Virgo interferometer.

These observational facilities have greatly advanced our understanding of various high-energy phenomena, including stellar-scale black holes in binary systems, supermassive black holes in Active Galactic Nuclei (AGNs), gamma-ray bursts (GRBs), a rich list of objects in a neutron star zoo, as well as supernovae and their remnants. The next 10 years will see a continued growth of the field, as technology has allowed several most challenging detectors to meet the sensitivity to potentially 
detect signals hitherto only speculated about. These include high-energy (above $10 \mathrm{TeV}$ ) neutrinos and gravitational waves. It is foreseen that the field of high energy astrophysics will fully embrace its "multi-messenger" nature as commonly envisioned.

This Special Issue of Frontiers of Physics is intended to provide a comprehensive overview of this exciting field. Reviews of various individual topics are available from many sources. However, there is no single source where interested readers can find an overview of all the directions in this field. This issue is aimed at filling this gap. The original plan was to publish 12 articles in 3 parts. It turned out that one review article (supernovae and their remnants) could not be finished in time, so the current issue only includes 11 articles.

The first part of the Special Issue is designed to survey the field according to high energy objects or phenomena. It includes active galactic nuclei (AGNs, H. Krawczynski and E. Treister) [1] that host super-massive black holes, stellar-size black hole X-ray binaries (BHXBs, S.N. Zhang) [2], gamma-ray bursts (GRBs, N. Gehrels and S. Razzaque) [3], and various species in the neutron star (NS) zoo (A. K. Harding) [4]. The 5th planned article on supernovae and their remnants did not materialize.

In Ref. [1], Krawzynski and Treister review the astrophysics of AGNs, the nuclei of galaxies that host supermassive black holes in the mass range $10^{6}-10^{10} M_{\odot}$, which accrete materials from the environment. In some cases, these accreting BHs launch a pair of powerful jets. Depending on the viewing angle to these systems and to a lesser degree on the intrinsic properties as well, AGNs display themselves in a rich trove of phenomenology. These are reviewed along with the underlying physics of jets and central engine, as well as evolution of AGNs within the cosmological context.

In Ref. [2], S.-N. Zhang surveys our current understanding of black hole X-ray binaries (BHXBs). These systems include a stellar-mass black hole, typically with a mass $3-20 M_{\odot}$, and a companion star from which matter is accreted towards the black hole $(\mathrm{BH})$ and form an accretion disk which is hot enough to emit X-rays. Jets are often associated with these systems, so these objects are also termed as "micro-quasars" by analogy with AGNs. The phenomenology and underlying physics are reviewed, with the focus on several important subjects, such as BH spin measurements, hot accretion flows and corona physics, as well as transition between two accretion states.
In the extreme of stellar-scale high-energy events, GRBs distinguish themselves as the most luminous explosions in the universe, which signal the birth of stellarmass black holes or rapidly spinning highly-magnetized neutron stars (NSs). This subject is reviewed by Gehrels and Razzaque [3], with the focus on the recent observational breakthroughs led by the NASA missions Swift and Fermi. These observations have greatly advanced our understanding of the physics of the GRB phenomenology, including the origin of the prompt gamma-ray emission and afterglow, as well as the physics of the central engine and the progenitor star of GRBs.

Besides BHs, another type of objects that high energy astrophysics studies extensively are neutron stars. These objects are believed to be composed of matter whose density is close to the nuclear density but whose interior composition remains unknown. There is a diverse population of NSs. In Ref. [4], Harding presents a comprehensive review of the observational and physical properties of the different species in the NS zoo, as well as the physical origins of the diversity. The objects surveyed include rotation-powered pulsars, magnetars, compact central objects, isolated neutron stars, and accreting neutron stars.

The second part of the Special Issue surveys the field of high energy astrophysics by means of the detection methods. There are many detectors that cover different observational channels (as summarized in the 2nd paragraph). It is impossible to review all of them here. With the focus on the highest energy and non-electromagnetic channels, we selected the following 5 topics: $\sim \mathrm{GeV} \gamma$ ray astronomy examplified by Fermi (S. Ritz) [5]; TeV $\gamma$-ray astronomy (F. M. Rieger, E. de Ona-Wilhelmi, and F. A. Aharonian) [6]; high energy cosmic ray astronomy (T. K. Gaisser, T. Stanev, and S. Tilav) [7]; high energy neutrino astronomy (F. Halzen) [8]; and gravitational wave astronomy (G. Gonzalez, A. Viceré, and L. Wen) [9].

The Fermi satellite has been extremely successful in unveiling the high energy universe from $30 \mathrm{MeV}$ to $>300$ GeV. In Ref. [5], Ritz summarizes the achievements of Fermi in various disciplines of astrophysics, including pulsars and nebulae, AGNs, GRBs, the "Fermi bubbles", and the $\gamma$-ray background. The endeavors of using Fermi data to shed light on fundamental physics problems, such as indirect search for dark matter signals as well as constraints on Lorentz Invariance Violation, are also highlighted. 
The $\mathrm{TeV}$ astronomy domain was recently rapidly advanced thanks to the successful completion of several ground-based detectors, such as H.E.S.S. and VERITAS. The exciting discoveries at $\mathrm{TeV}$ energies are reviewed by Rieger et al. [6] along with their astrophysical implications.

Compared with other branches, cosmic ray astronomy may be the earliest branch in high energy astrophysics. Yet, the energy spectra of all particles and different species are still not measured precisely, and their physical origins remain elusive. In Ref. [7], Gaisser et al. critically review this field, paying special attention to the energy spectrum in the $\mathrm{PeV}$ range and above. They present a baseline spectrum from $10^{14}$ to $10^{20} \mathrm{eV}$ obtained by combining the measurements published in various sources, and they discuss the astrophysical implications of the results.

High energy neutrinos are believed to be produced through hadronic interaction processes from astronomical sources that accelerate high energy cosmic rays. Detecting them is extremely challenging. In Ref. [8], Halzen reviews the journey of constructing the first kilometerscale neutrino detector IceCube in Antarctica, discussing the astrophysical motivations to construct such a detector, as well as the prospects to detect high energy neutrinos from astrophysical sources in the near future.

In another direction, González et al. [9] show that gravitational wave astronomy is entering a new era in which the very first direct detection of gravitational waves becomes possible. They review various astrophysical sources that are likely gravitational wave emitters, and show how the current technology is getting close to reaching the sensitivity to detect these faint signals.

The Special Issue ends with the third part that includes two reviews on two important subjects of the contemporary astrophysics, namely, dark matter (X.-J. Bi, P.-F. Chen, and Q. Yuan) [10] and dark energy (M. Li, X.-D. Li, S. Wang, and Y. Wang) [11]. These two subjects are not limited to high energy astrophysics, having a large impact on astrophysics as whole (especially cosmology), as well as being major problems of fundamental physics. Nonetheless, they are closely connected to high energy astrophysics. For example, observations of electron/positron cosmic rays, $\gamma$-ray lines, and neutrinos may lead to indirect detection of dark matter. The existence of dark energy was first revealed by observing the space distribution of explosions of high-energy events Type Ia supernovae, and the nature of dark energy may be revealed by combining measurements of different standard candles, including those in high energy phenomena such as GRBs.

In Ref. [10], Bi et al. review the status and progress in dark matter (DM) searches, including direct searches of recoils and ionization from DM-kicks to standard model (SM) particles in underground experiments, collider searches of missing energy carried by DM particles, as well as indirect searches of $\gamma$-rays and leptonic cosmic rays due to DM-DM interactions in astrophysical environments. Even though no robust detection is available yet, they show several interesting observations that may suggest evidence of DM, and they discuss the promises and issues of these observations.

Finally, Li et al. [11] review various astrophysical lines of evidence for the existence of dark energy (DE), and an impressive list of theoretical particle physics models that interpret the DE phenomenon. They also discuss how the theoretical models are constrained by the available and future astrophysical data.

We are lucky to live in a special epoch in the human history. Observations have allowed us to measure the parameters of our universe with increasing precision, and to infer the composition of the universe we are living in. Ground-breaking discoveries over the decades have extended our horizon to understand the universe, from the brightest (e.g. GRBs, AGN blazars) to the darkest (e.g. dark matter and dark energy). More excitingly, we are on the technological brink of observing the universe using brand new observational channels, such as high energy neutrinos and gravitational waves. For the first time in history, the next decade will probably witness the first truly multi-messenger observational campaign of a high energy phenomenon. Theoretical modeling will be advanced to address these new discoveries, with many open questions answered or partially addressed. Numerical simulations will continue to ripen by combining general relativity and resistive MHD at the macroscopic level and particle-in-cell simulations at the microscopic level. More advanced computers and algorithms will allow simulations to unprecedented levels of accuracy to address fundamental questions such as magnetospheric structure, jet launching, energy dissipation, particle acceleration and radiation in high energy astronomical systems. If history is any guide, it is foreseen that new observations will raise more questions and challenges, which will give continuous impetus for the further development of new technologies and theoretical ideas to better understand 
the exciting and mysterious universe we are living in.

Finally, we would like to thank all the invited reviewers of this Special Issue, without whose dedication and great efforts this volume would not be possible. We also thank Dr. Hong-Guang Dong for restlessly providing editorial support to the authors.

\section{References}

1. H. Krawczynski and E. Treister, Front. Phys., 2013, 8(6): 609, arXiv: 1301.4179

2. S.-N. Zhang, Front. Phys., 2013, 8(6): 630, arXiv: 1302.5485

3. N. Gehrels and S. Razzaque, Front. Phys., 2013, 8(6): 661, arXiv: 1301.0840
4. A. K. Harding, Front. Phys., 2013, 8(6): 679, arXiv: 1302.0869

5. S. Ritz, Front. Phys., 2013, 8(6): 693

6. F. M. Rieger, E. de Oña-Wilhelmi, and F. A. Aharonian, Front. Phys., 2013, 8(6): 714, arXiv: 1302.5603

7. T. K. Gaisser, T. Stanev, and S. Tilav, Front. Phys., 2013, 8(6): 748, arXiv: 1303.3565

8. F. Halzen, Front. Phys., 2013, 8(6): 759

9. G. González, A. Viceré, and L. Wen, Front. Phys., 2013, 8(6): 771

10. X.-J. Bi, P.-F. Yin, and Q. Yuan, Front. Phys. 2013, 8(6): 794

11. M. Li, X.-D. Li, S. Wang, and Y. Wang, Front. Phys., 2013, 8(6): 828 , arXiv: 1209.0922 